\title{
A Docência do Ensino Superior em saúde em tempos de pandemia da Covid-19
}

\author{
Higher Education Teaching in health at Covid-19 pandemic times \\ Enseñanza de la Educación Superior en salud en tiempos de pandemia de Covid-19
}

Nanielle Silva Barbosa

ORCID: https://orcid.org/0000-0001-5758-2011 Universidade Estadual do Piauí, Brasil

E-mail: naniellesilvabarbosa@ hotmail.com

Kayron Rodrigo Ferreira Cunha ORCID: https://orcid.org/0000-0003-3507-3376 Universidade Federal do Piauí, Brasil E-mail: ikayron.kr@gmail.com

Maria Samara Da Silva

ORCID: https://orcid.org/0000-0001-6987-1224 Universidade Estadual do Piauí, Brasil E-mail: mariasamara2v@gmail.com

Amanda Fernanda Damasceno Saraiva de Sousa ORCID: https://orcid.org/0000-0003-2395-8353 Universidade Federal do Piauí, Brasil E-mail: amandamascpsi@gmail.com

Francisca Aline Amaral da Silva ORCID: https://orcid.org/0000-0003-4931-808X Universidade Estadual do Piauí, Brasil E-mail: franciscaaline@ccs.uespi.br

Luciana Kelly da Silva Fonseca ORCID: https://orcid.org/0000-0001-8832-5261 Universidade Federal do Piauí, Brasil E-mail: 1.kelly_fonseca@ hotmail.com

Karolaine Rodrigues Louzeiro ORCID: https://orcid.org/0000-0002-5728-2657 Centro Universitário UNINOVAFAPI, Brasil E-mail: karolainne97@outlook.com

Thiemmy de Souza Almeida Guedes ORCID: https://orcid.org/0000-0003-2261-0320 Faculdade Venda Nova do Imigrante-FAVENI, Brasil E-mail: thiemmyalmeida@gmail.com

Rosilene Maria do Espirito Santo Carvalho ORCID: https://orcid.org/0000-0003-1770-9158 Faculdade UNINASSAU, Brasil E-mail: rosilenemaria20@hotmail.com

Kyvia Naysis de Araujo Santos ORCID: https://orcid.org/0000-0003-3707-986X Universidade Federal do Piauí, Brasil

E-mail: kyvianaysis@gmail.com

Cristiana Pacífico Oliveira

ORCID: https://orcid.org/0000-0002-7094-3333 Universidade Federal do Piauí, Brasil E-mail: cris.enferm@hotmail.com

Larissa da Silva Sampaio ORCID: https://orcid.org/0000-0001-6381-6515

Universidade Federal do Maranhão, Brasil E-mail: sampaioslarissa@gmail.com Ana Raquel Rodrigues Rosa

ORCID: https://orcid.org/0000-0003-2285-6582 Universidade Federal do Piauí, Brasil E-mail: anaraquelrrr21@ hotmail.com

Nathaly Marques Santos

ORCID: https://orcid.org/0000-0001-8737-423X Universidade Federal do Piauí, Brasil

Amanda Karoliny Meneses Resende ORCID: https://orcid.org/0000-0001-7414-999X Universidade Federal do Piauí, Brasil E-mail: amandakaroliny.10@gmail.com 


\begin{abstract}
Resumo
Este estudo traz como objetivo discutir o cenário da Docência do Ensino Superior na área da saúde em tempos de pandemia da Covid-19. Trata-se de uma revisão integrativa com busca realizada nos meses de maio a julho de 2021 nas bases de dados: Literatura Latino-Americana e do Caribe em Ciências da Saúde, Base de Dados de Enfermagem, Índice Bibliográfico Espanhol em Ciências da Saúde, Medical Literature Analysis and Retrieval System Online e Scientific Eletronic Library Online. Para a busca foram utilizados os descritores: "docência", "ensino", e "coronavírus". Foram definidos como critérios de inclusão: artigos elaborados com base em estudos primários, publicados nos idiomas inglês, português e espanhol, relacionados à temática investigada e sem delimitação de recorte temporal. Quinze produções foram incluídas para síntese. A análise crítica e aprofundada possibilitou a organização dos resultados por similaridade semântica, o que permitiu a construção de duas categorias temáticas: um novo modelo de ensino-aprendizagem e o uso das tecnologias educacionais e educação a distância emergencial: barreiras e facilitadores. Infere-se que o atual cenário de saúde pública exige das Instituições de Ensino Superior um processo de transformação, adaptação e exercício da criatividade para lidar com o ensino remoto emergencial e o uso das tecnologias digitais.
\end{abstract}

Palavras-chave: Pandemias; Coronavírus; Ensino; Tecnologias educacionais.

\begin{abstract}
This study aims to discuss the scenario of Higher Education Teaching in the area of health in times of pandemic of Covid-19. This is an integrative review with a search carried out from May to July 2021 in the following databases: Latin American and Caribbean Literature in Health Sciences, Nursing Database, Spanish Bibliographic Index in Health Sciences, Medical Literature Analysis and Retrieval System Online and Scientific Electronic Library Online. For the search, the following descriptors were used: 'teaching', 'education' and 'coronavirus'. The inclusion criteria were defined as: articles based on primary studies, published in English, Portuguese and Spanish, related to the investigated theme and without a time frame. Fifteen productions were included for synthesis. The critical and indepth analysis made it possible to organize the results by semantic similarity, which allowed the construction of two thematic categories: a new teaching-learning model and the use of educational technologies and emergency distance education: barriers and facilitators. It is inferred that the current public health scenario requires from Higher Education Institutions a process of transformation, adaptation and exercise of creativity to deal with emergency remote education and the use of digital technologies.
\end{abstract}

Keywords: Pandemics; Coronavirus; Teaching; Educational technologies.

\title{
Resumen
}

Este estudio tiene como objetivo discutir el escenario de la Docencia de Educación Superior en el área de la salud en tiempos de pandemia de Covid-19. Se trata de una revisión integradora con una búsqueda realizada de mayo a julio de 2021 en las siguientes bases de datos: Literatura Latinoamericana y del Caribe en Ciencias de la Salud, Base de Datos de Enfermería, Índice Bibliográfico Español en Ciencias de la Salud, Sistema de Análisis y Recuperación de Literatura Médica en Línea y Biblioteca Electrónica Científica en Línea. Para la búsqueda se utilizaron los siguientes descriptores: 'enseñanza', 'educación' y 'coronavirus'. Los criterios de inclusión se definieron como: artículos basados en estudios primarios, publicados en inglés, portugués y español, relacionados con el tema investigado y sin marco temporal. Se incluyeron quince producciones para síntesis. El análisis crítico y en profundidad permitió organizar los resultados por semejanza semántica, lo que permitió la construcción de dos categorías temáticas: un nuevo modelo de enseñanza-aprendizaje y el uso de tecnologías educativas y educación a distancia de emergencia: barreras y facilitadores. Se infiere que el actual escenario de salud pública requiere de las Instituciones de Educación Superior un proceso de transformación, adecuación y ejercicio de la creatividad para enfrentar la educación remota de emergencia y el uso de tecnologías digitales.

Palabras clave: Pandemias; Coronavirus; Enseñanza; Tecnologías educativas.

\section{Introdução}

O vírus SARS-COV-2, nome que recebeu o patógeno causador da covid-19, foi o responsável por uma série de casos de uma pneumonia atípica em Wuhan, cidade localizada na China, ainda em meados de dezembro de 2019. Esses casos relacionados ao vírus rapidamente passaram de um surto isolado a uma emergência de saúde pública a nível global. Em 11 de março de 2020 a Organização Mundial de Saúde (OMS) declarou, o momento desencadeado pelo aumento da contaminação, situação de calamidade pública, instalando o status de pandemia (Mojica \& Morales, 2020).

Há relatos de que o vírus chegou na América Latina em 25 de fevereiro de 2020, quando o Ministério da Saúde do Brasil confirmou o primeiro caso da doença. Até o dia 02 de agosto de 2021, o país havia atingido 19.938 .358 de casos 
confirmados e 556.834 óbitos. Enquanto isso ocorria no mundo, um aumento no número de casos e mortes, chegando a 198.614.523 pessoas contaminadas com 4.229.142 óbitos (Cespedes \& Souza, 2020; Brasil, 2021).

A pandemia da covid-19 ocasionou e ocasiona repercussões intensas na situação de saúde das pessoas, em virtude do seu potencial de disseminação. Além disso, tem impactado em outros cenários, a exemplo, o educacional. Tal impacto perpassa por um processo de adequação da formação acadêmica nas universidades, inclusive em áreas da saúde (Brasil, 2020).

A suspensão temporária das aulas presenciais tem sido uma das estratégias adotadas na tentativa de reduzir o risco de contágio e proliferação do vírus entre os atores da educação, professores, alunos e demais funcionários, exigindo a adequação do sistema de ensino a uma nova realidade. No Brasil, inúmeras instituições de ensino públicas e privadas tiveram suas atividades presenciais suspensas atendendo a Portaria n 343 de 17 de março de 2020 e a Medida Provisória no 934 de 1 de abril de 2020 (Castaman \& Rodrigues, 2020).

O fechamento das escolas básicas e instituições de ensino superior trouxe um desafio inédito à educação mundial. Diante disso, para manter a relação entre alunos e professores e a fim de tentar minimizar o prejuízo no ensino, muitas instituições ao redor do mundo estão sendo obrigadas a implementar e inovar suas metodologias de aprendizado, incorporando, por exemplo, as tecnologias digitais. No entanto, o que preocupa é que o acesso a essa modalidade não é igualitária em todas as regiões do globo, considerando que nem todas as pessoas possuem a disponibilidade de internet e equipamentos para tal, principalmente em locais mais vulneráveis (The World Bank, 2020; Carvalho, Lima \& Coeli, 2020).

A partir desse contexto, o interesse pela discussão da temática justifica-se a partir das vivências no âmbito ensinopedagógico vivenciado pelos autores, que por atuarem como docentes, atividade relacionada a carga horária dentro de um programa de residência multiprofissional em saúde, perceberam em seu dia-a-dia as dificuldades em se adaptar a um novo cenário de ensino-aprendizagem, incluindo barreiras, limitações e queixas de seus próprios alunos.

Este estudo destaca-se como relevante à medida que apresenta a comunidade cientifica evidências ainda restritas e limitadas, indicando a necessidade de expansão dos estudos e pesquisas relacionadas ao tema com o intuito de contribuir para a reflexão de situações-problemas e fomentar propostas para seu enfrentamento. Logo, traz como objetivo discutir, com base em evidências científicas, o cenário da Docência do Ensino Superior na área da saúde em tempos de pandemia da Covid-19.

\section{Metodologia}

Este estudo trata-se de uma revisão integrativa da literatura construída conforme seis etapas recomendadas para sua elaboração: identificação do tema e elaboração da questão de pesquisa; estabelecimento de critérios para inclusão e exclusão de estudos/ amostragem ou busca na literatura; definição das informações a serem extraídas dos estudos selecionados/ categorização dos estudos; avaliação dos estudos incluídos na revisão integrativa; interpretação dos resultados; apresentação da revisão/síntese do conhecimento (Mendes, Silveira \& Galvão, 2008).

Por meio da revisão integrativa é possível incluir e analisar evidencias cientificas relevantes que contribuem para a tomada de decisão e a melhoria da prática clínica a medida que permite um conhecimento aprofundado sobre determinado assunto, bem como apontar lacunas a serem preenchidas com realização de novos estudos. Para isso é necessário seguir uma metodologia rigorosa que forneça clareza nos resultados apresentados (Sousa et al., 2020).

Para a elaboração da questão norteadora tomou-se como base o acrônimo PICo, definindo-se: P (população/problema) = "docentes"/" "docência", I (fenômeno de interesse): "docência do ensino superior em saúde", Co (contexto) = pandemia da Covid-19. Assim, formulou-se a seguinte questão de pesquisa: como encontra-se o cenário da docência do ensino superior em saúde durante a pandemia da Covid-19?

O levantamento bibliográfico foi realizado durante os meses de maio a julho de 2021, o recorte temporal dos artigos analisados se deu entre janeiro de 2020 a junho de 2021, considerando o período correspondente a pandemia da Covid-19, nas 
bases eletrônicas de dados: Literatura Latino-Americana e do Caribe em Ciências da Saúde (LILACS), Base de Dados de Enfermagem (BDENF), Índice Bibliográfico Espanhol em Ciências da Saúde (IBECS), Medical Literature Analysis and Retrieval System Online (MEDLINE) e Scientific Eletronic Library Online (SciELO). Para a busca foram utilizados os descritores: "docência", "ensino" e "coronavírus", consultados no Medical Subject Headings (MeSH) e Descritores em Ciências da Saúde (DeCS), combinados com o operador boleano AND.

Foram definidos como critérios de inclusão: artigos elaborados com base em estudos primários, publicados nos idiomas inglês, português e espanhol, relacionados à temática investigada e sem delimitação de recorte temporal. Excluíram-se notas, monografias, dissertações e teses.

Com o intuito de minimizar prováveis erros ou vieses de aferição dos estudos, a seleção foi realizada de forma independente, por dois revisores, em duas etapas. Na primeira etapa, realizou-se a leitura de título e resumo e, na segunda, fezse a leitura do texto completo. Nos casos em que ocorreram desacordos, houve discussão entre os dois avaliadores para alcançar um consenso. A seguir, fluxograma que descreve as etapas decorridas para a seleção dos artigos (Figura 1):

Figura 1 - Fluxograma de identificação, seleção, elegibilidade e inclusão dos artigos.

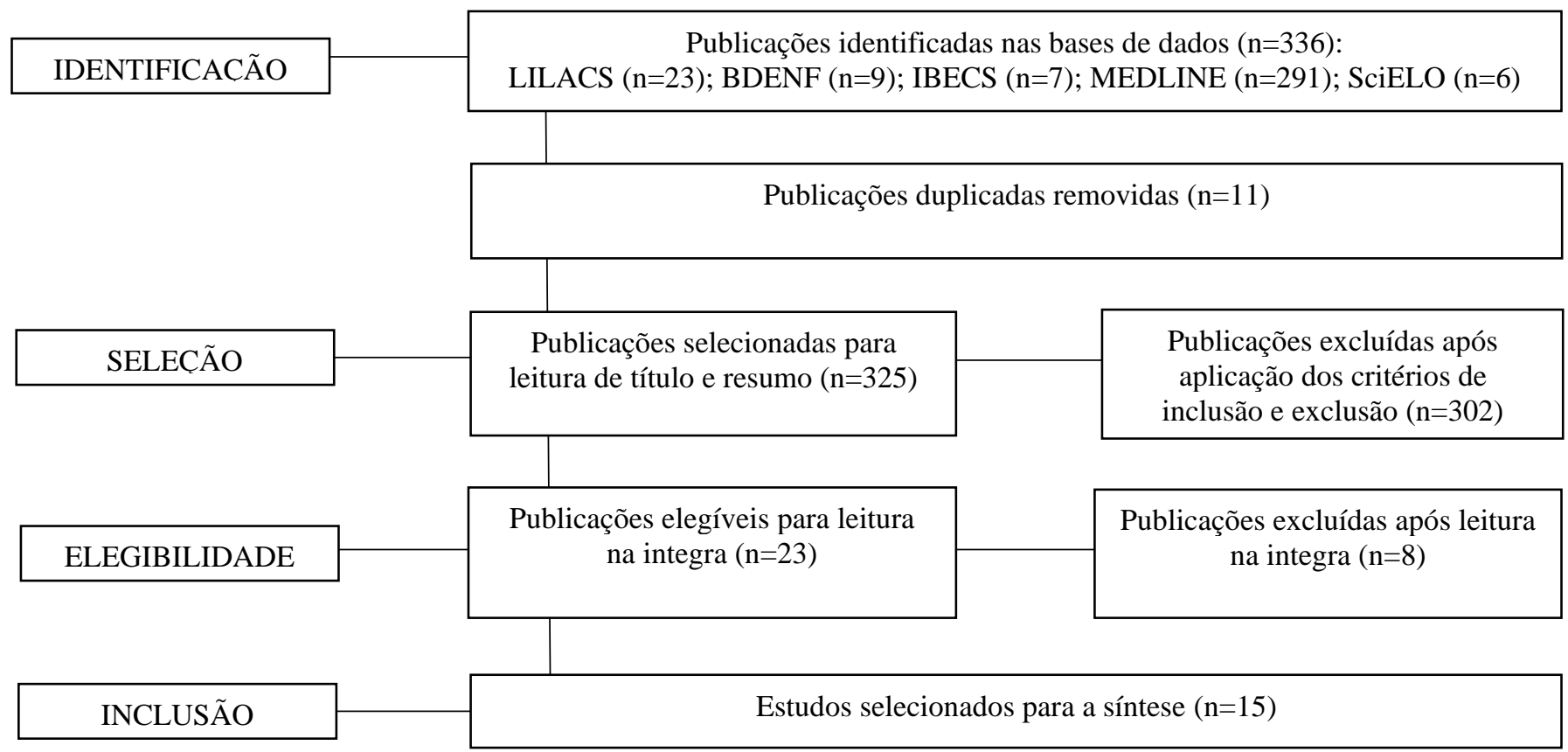

Fonte: Elaborado pelo autor a partir do Fluxograma PRISMA (2021).

Para a extração de dados considerados relevantes em cada produção os autores elaboraram formulário contendo informações sobre identificação do artigo, autoria, país e ano de publicação, abordagem metodológica, principais conclusões e nível de evidência (NE). As evidências foram classificadas conforme o modelo proposto: nível I (evidências oriundas de revisão sistemática ou metanálise de todos ensaios clínicos relevantes randomizados controlados ou provenientes de diretrizes clínicas baseadas em revisões sistemáticas de ensaios clínicos randomizados controlados); nível II (evidências derivadas de pelo menos um ensaio clínico randomizado controlado bem delineado); nível III (evidências obtidas de ensaios clínicos bem delineados sem randomização); nível IV (evidências provenientes de estudos de coorte e de caso-controle bem delineados); nível V (evidências originárias de revisão sistemática de estudos descritivos e qualitativos); nível VI (evidências derivadas de um único estudo descritivo ou qualitativo); e nível VII (evidências oriundas de opinião de autoridades e/ou relatórios de comitês de especialistas) (Melnyk \& Fineout-Overholt, 2005). 


\section{Resultados e Discussão}

Quinze produções (100\%) foram incluídas para síntese. Dez artigos (66,6\%) foram publicados no ano de 2021, ressaltando a abordagem recente na literatura sobre o tema. Houve predomínio de publicações internacionais, 12 (80\%). Quanto ao NE, as 15 produções (100\%) classificaram como nível VI (evidências derivadas de um único estudo descritivo ou qualitativo). Os estudos evidenciaram a utilização das tecnologias educacionais, benefícios e limitações durante a pandemia da Covid-19. A síntese dos achados encontra-se organizada na Tabela 1.

Tabela 1 - Sumarização das produções incluídas na síntese conforme título, autores, ano, país de publicação, abordagem metodológica, principais conclusões e NE.

\begin{tabular}{|c|c|c|c|c|}
\hline Título & $\begin{array}{c}\text { Autores/ } \\
\text { Ano/País de } \\
\text { publicação }\end{array}$ & Abordagem metodológica & Principais conclusões & NE \\
\hline $\begin{array}{lrr}\text { Ensino } & \text { remoto } & \text { emergencial } \\
\text { na graduação } & \text { em } \\
\text { enfermagem: relato } & \text { de } \\
\text { experiência na covid-19 } & \end{array}$ & $\begin{array}{l}\text { Bastos et al., } \\
\text { 2020/Brasil }\end{array}$ & $\begin{array}{l}\text { Tipo de estudo: relato de experiência } \\
\text { Cenário: Centro Universitário Período: } \\
\text { março a maio de } 2020 \\
\begin{array}{lll}\text { Amostra: } 1100 \quad \text { estudantes } \quad \text { e } \quad 40 \\
\text { professores } \\
\text { Instrumentos: não especificado }\end{array} \\
\end{array}$ & $\begin{array}{l}\text { É necessário readequação das estratégias de } \\
\text { ensino, as quais variaram desde a realização } \\
\text { de conferências virtuais até a elaboração } \\
\text { conjunta de produtos que compuseram } \\
\text { atividades avaliativas nesse ambiente. }\end{array}$ & VI \\
\hline $\begin{array}{l}\text { Estratégias de ensino à } \\
\text { distância para a educação } \\
\text { interprofissional em saúde } \\
\text { frente à pandemia COVID-19 }\end{array}$ & $\begin{array}{l}\text { Granjeiro et al., } \\
\text { 2020/Brasil }\end{array}$ & $\begin{array}{l}\text { Tipo de estudo: relato de experiência } \\
\text { Cenário: Centro de Atendimento ao } \\
\text { Diabético e Hipertenso (CADH) } \\
\text { Periodo: março a junho de } 2020 \\
\text { Amostra: seis discentes e sete docentes } \\
\text { Instrumentos: não especificado }\end{array}$ & $\begin{array}{l}\text { A implementação de atividades utilizando } \\
\text { plataformas virtuais foram fundamentais } \\
\text { para o planejamento do conjunto de ações } \\
\text { realizadas, culminando no ganho de } \\
\text { conhecimento individual e coletivo; } \\
\text { implementação de alterações na rotina do } \\
\text { serviço e orientação aos pacientes e } \\
\text { familiares sobre a pandemia. }\end{array}$ & VI \\
\hline $\begin{array}{lll}\text { Estratégias } & \text { e desafios } & \text { do } \\
\text { ensino } & \text { remoto } & \text { na } \\
\text { enfermagem } & & \\
\end{array}$ & $\begin{array}{l}\text { Silveira et al., 2020/ } \\
\text { Brasil. }\end{array}$ & $\begin{array}{l}\text { Tipo de estudo: relato de experiência } \\
\text { Cenário: Instituições de Ensino } \\
\text { Superior } \\
\text { Período: março de } 2020 \\
\text { Amostra: seis docentes } \\
\text { Instrumentos: não especificado }\end{array}$ & $\begin{array}{l}\text { Repensar novas práticas de ensino e } \\
\text { aprendizagem na educação superior é um } \\
\text { desafio para os docentes do curso de } \\
\text { Enfermagem no que tange a reorganização } \\
\text { de seus planos de ensino para que possam } \\
\text { atingir o objetivo da educação remota e } \\
\text { inclusão de ferramentas tecnológicas. }\end{array}$ & VI \\
\hline $\begin{array}{l}\text { Innovación emergente para la } \\
\text { COVID-19: taller virtual } \\
\text { sobre el uso educativo de la } \\
\text { plataforma Moodle }\end{array}$ & $\begin{array}{l}\text { Bory, Flores \& } \\
\text { Díaz, 2021/ Cuba }\end{array}$ & $\begin{array}{l}\text { Tipo de estudo: relato de experiência } \\
\text { Cenário: Instituição de Ensino Superior } \\
\text { Período: não especificado } \\
\text { Amostra: não especificada } \\
\text { Instrumentos: não especificado }\end{array}$ & $\begin{array}{l}\text { A intervenção estratégica para a formação de } \\
\text { professores no uso educacional } \\
\text { da plataforma Moodle tem importância } \\
\text { social para lançar as bases para iniciar um } \\
\text { processo de ensino organizacional inovador. }\end{array}$ & VI \\
\hline 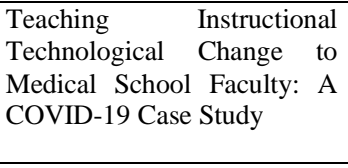 & $\begin{array}{l}\text { Cote et al., 2020/ } \\
\text { Estados Unidos da } \\
\text { America }\end{array}$ & $\begin{array}{l}\text { Tipo de estudo: estudo de caso } \\
\text { Cenário: Istituição de Ensino Superior } \\
\text { Período: não especificado } \\
\text { Amostra: não especificado } \\
\text { Instrumentos: questionários online }\end{array}$ & $\begin{array}{l}\text { A capacitação oferecida pela plataforma } \\
\text { permitiu maior reconhecimento de sua } \\
\text { importância por parte dos professores já que } \\
\text { houve a preocupação em orienta-los como } \\
\text { utilizar os recursos digitais educacionais. }\end{array}$ & VI \\
\hline $\begin{array}{l}\text { Gross Anatomy Education in } \\
\text { China during the Covid-19 } \\
\text { Pandemic: A National } \\
\text { Survey }\end{array}$ & $\begin{array}{l}\text { Cheng et al., } \\
\text { 2021/China. }\end{array}$ & $\begin{array}{l}\text { Tipo de estudo: descritivo, quantitativo } \\
\text { Cenário: Instituições de Ensino } \\
\text { Superior } \\
\text { Período: não especificado } \\
\text { Amostra: } 359 \text { docentes } \\
\text { Instrumentos: questionário online }\end{array}$ & $\begin{array}{l}\text { A pesquisa constatou que um número } \\
\text { significativo de escolas médicas na China já } \\
\text { tinha experiência em ensino online e } \\
\text { infraestrutura que possibilitou a rápida } \\
\text { implantação do aprendizado totalmente } \\
\text { online. Por outro lado, algumas escolas } \\
\text { optaram por suspender suas sessões práticas } \\
\text { durante a pandemia. As dificuldades } \\
\text { descritas pelos professores nesta pesquisa } \\
\text { fornecem uma visão sobre os obstáculos ao } \\
\text { desenvolvimento da educação em anatomia } \\
\text { macroscópica na China. Esses obstáculos } \\
\text { existiam anteriormente, mas foram } \\
\text { descobertos por esta pandemia. }\end{array}$ & VI \\
\hline $\begin{array}{l}\text { Undergraduate medical } \\
\text { education amid COVID-19: a } \\
\text { qualitative analysis of } \\
\text { enablers and barriers to } \\
\text { acquiring competencies in } \\
\text { distant learning using focus } \\
\text { groups }\end{array}$ & $\begin{array}{l}\text { Reinhart et al., } \\
\text { 2021/Alemanha }\end{array}$ & $\begin{array}{l}\text { Tipo de estudo: relato de experiência } \\
\text { Cenário: Instituição de Ensino Superior } \\
\text { Período: não especificado } \\
\text { Amostra: } 193 \text { discente e } 32 \text { professores } \\
\text { Instrumentos: não especificado }\end{array}$ & $\begin{array}{l}\text { A intervenção pode servir para uma } \\
\text { transição cuidadosa e necessária para o } \\
\text { futuro } e \text {-learning } \\
\text { e programas híbridos para uma educação } \\
\text { médica baseada em competências com } \\
\text { medidas contínuas de distanciamento social. }\end{array}$ & VI \\
\hline $\begin{array}{l}\text { COVID-19's impact on } \\
\text { faculty and staff at a School } \\
\text { of Medicine in the US: what } \\
\text { is the blueprint for the }\end{array}$ & $\begin{array}{l}\text { Gottenborg et al., } \\
\text { 2021/Estados } \\
\text { Unidos da América }\end{array}$ & $\begin{array}{l}\text { Tipo de estudo: qualitativo } \\
\text { Cenário: Instituição de Ensino Superior } \\
\text { Período: julho a agosto de } 2020 \\
\text { Amostra: } 28 \text { professores e funcionários }\end{array}$ & $\begin{array}{l}\text { Ambos os grupos alunos e instrutores, } \\
\text { ficaram satisfeitos com a rápida transição } \\
\text { para o ensino à distância } \\
\text { devido ao COVID-19. Essa transição trouxe }\end{array}$ & VI \\
\hline
\end{tabular}




\begin{tabular}{llll}
\hline future? & & \\
\hline Rapid transition to distance & Rad et al., \\
learning due to COVID-19: & 2021/Emirados \\
Perceptions of postgraduate & Árabes Unidos \\
dental learners and & \\
instructors & &
\end{tabular}

Instrumentos: questionário online

Tipo de estudo: descritivo, quanti qualitativo

Cenário: Instituição de Ensino Superior

Período: maio de 2020

Amostra: 63 estudantes e 21 docentes

Instrumentos: questionário online vantagens e desafios.

A transição abrupta para o ensino a distância, devido ao COVID-19, foi percebida favoravelmente. Essa mudança inesperada envolveu a superação de muitos desafios, mas também revelou oportunidades substanciais que valem a pena capitalizar no aprendizado e ensino dos profissionais de saúde.

\begin{tabular}{|c|c|c|c|c|}
\hline $\begin{array}{l}\text { Perception of medical } \\
\text { education by learners and } \\
\text { teachers during the COVID- } \\
19 \text { pandemic: } \\
\text { crosssectional survey of } \\
\text { online teaching }\end{array}$ & $\begin{array}{l}\text { Motte-Signoret et } \\
\text { al., 2021/ França. }\end{array}$ & $\begin{array}{l}\text { Tipo de estudo: descritivo, transversal } \\
\text { Cenário: Instituição de Ensino Superior } \\
\text { Período: junho a agosto } 2020 \\
\begin{array}{ll}\text { Amostra: } 146 \quad \text { estudantes e } 26 \\
\text { professores }\end{array} \\
\text { Instrumentos: questionário online } \\
\end{array}$ & $\begin{array}{l}\mathrm{O} \text { ensino online oferecido é uma forma } \\
\text { apropriada de ensino durante a pandemia. }\end{array}$ & VI \\
\hline $\begin{array}{l}\text { The perceptions of anatomy } \\
\text { teachers for different majors } \\
\text { during the COVID-19 } \\
\text { pandemic: a national Chinese } \\
\text { survey }\end{array}$ & $\begin{array}{l}\text { Yan et } \\
2021 / \text { China. }\end{array}$ & $\begin{array}{l}\text { Tipo de estudo: pesquisa exploratória, } \\
\text { descritiva, qualitativa } \\
\text { Cenário: Instituição de Ensino Superior } \\
\text { Período: fevereiro a abril de } 2020 \\
\text { Amostra: } 366 \text { professores } \\
\text { Instrumentos: } \\
\text { questionário online }\end{array}$ & $\begin{array}{l}\text { Em relação aos maiores ganhos e } \\
\text { dificuldades, os professores acreditavam que } \\
\text { o ensino online durante a pandemia } \\
\text { proporcionava uma boa oportunidade para } \\
\text { desenvolver soluções pedagogicamente } \\
\text { inovadoras para facilitar a aprendizagem. }\end{array}$ & VI \\
\hline
\end{tabular}
$\begin{array}{llllll}\text { Barriers and facilitators } & \mathrm{Li} \text { et } & \text { al., 2021/ } & \text { Tipo de estudo: pesquisa transversal } \\ \text { to online medical and nursing } & \text { China. }\end{array} \quad \begin{aligned} & \text { quantitativa Cenário: Instituição de }\end{aligned}$

education

during the COVID-19

pandemic: perspectives

from international students

from low- and middle-

income countries and their

teaching staf
A atual crise do COVID-19 afeta

significativamente o resultado da educação online de alunos e professores internacionais, ao passo que a influência pode estar na disparidade de acordo com a situação de pandemia em seus atuais locais de residência em diferentes países.
Período: abril de 2020

Amostra: 316 e 120 professores

Instrumentos: questionário online
Satisfaction with online

learning in the new normal:

perspective of students and

faculty at medical and health

sciences colleges

\section{Elshami et al., Tipo de estudo: pesquisa exploratória 2021/Emirados transversal, quantitativa Árabes Unidos \\ Cenário: Instituição de Ensino Superior \\ Período: abril e maio de 2020 \\ Amostra: 370 estudantes e 81 docentes \\ Instrumentos: questionário online}

Os desafios enfrentados pelos alunos eram a longa duração das sessões de aprendizagem e da tecnologia. Por outro lado, a assistência técnica e $\mathrm{o}$ entusiasmo dos alunos aumentaram a satisfação do corpo docente com o ensino online. Maior carga de trabalho, maior tempo de preparação e problemas técnicos foram desafios relatados pelo corpo docente.

\begin{tabular}{|c|c|c|c|c|}
\hline $\begin{array}{lr}\text { Training } & \text { Faculty as an } \\
\text { Institutional } & \text { Response to } \\
\text { COVID-19 } & \text { Emergency } \\
\text { Remote Teaching Supported } \\
\text { by Data }\end{array}$ & $\begin{array}{l}\text { Walsh et al., } \\
\text { 2021/Estados } \\
\text { Unidos da América }\end{array}$ & $\begin{array}{l}\text { Tipo de estudo: pesquisa quanti e } \\
\text { qualitativa } \\
\text { Cenário: Instituição de Ensino Superior } \\
\text { Período: não especificado } \\
\text { Amostra: } 113 \text { professores } \\
\text { Instrumentos: questionário online }\end{array}$ & $\begin{array}{l}\text { Professores não treinados } \\
\text { incluíram o envolvimento do aluno como } \\
\text { uma dificuldade e eram mais propensos a } \\
\text { descrever uma memória negativa e } \\
\text { desanimadora de experiências com o ensino } \\
\text { online. }\end{array}$ & VI \\
\hline $\begin{array}{l}\text { Opportunities and challenges } \\
\text { of e-learning in vocational } \\
\text { training in General Practice - } \\
\text { a project report about } \\
\text { implementing digital formats } \\
\text { in the KWBW- } \\
\text { Verbundweiterbildung }\end{array}$ & $\begin{array}{l}\text { Förster et al., 2020/ } \\
\text { Alemanha }\end{array}$ & $\begin{array}{l}\text { Tipo de estudo: pesquisa qualitativa } \\
\text { Cenário: Instituição de Ensino Superior } \\
\text { Período: não especificado } \\
\text { Amostra: } 101 \text { estagiários e professores } \\
\text { Instrumentos: questionário online }\end{array}$ & $\begin{array}{l}\text { Das principais vantagens mencionadas foram } \\
\text { a ausência de viagens, economia de tempo e } \\
\text { custos e maior flexibilidade com os } \\
\text { encontros on-line. As principais } \\
\text { desvantagens mencionadas foram menos } \\
\text { interação pessoal e obstáculos técnicos. }\end{array}$ & $\mathrm{I}$ \\
\hline
\end{tabular}

Fonte: Dados da Pesquisa (2021).

A análise crítica e aprofundada possibilitou a organização dos resultados por similaridade semântica, o que permitiu a construção de duas categorias temáticas: um novo modelo de ensino-aprendizagem e o uso das tecnologias educacionais e educação a distância emergencial: barreiras e facilitadores.

\subsection{Um novo modelo de ensino-aprendizagem e o uso das tecnologias educacionais}

A adoção do isolamento social foi uma das medidas adotadas para interromper a propagação do novo coronavírus, causador da Covid-19, em decorrência do cenário de emergência que a sociedade se encontra. O Ministério da Saúde define o isolamento como a ação que objetiva a separação de pessoas sintomáticas ou assintomáticas de maneira a conter a disseminação viral. Assim, essa medida tem sido adotada pelos países e ainda não se sabe exatamente quando deixará de ser necessária (Brasil, 2020; Cespedes \& Souza, 2020). 
As medidas adotadas para o controle da pandemia da Covid-19 impactaram em diversos sistemas, não excluindo o educacional. Segundo a Organização das Nações Unidas para a Educação, a Ciência e a Cultura (UNESCO), mais de 1,3 bilhão de alunos de todo o mundo estão sendo afetados pelo fechamento das escolas e universidades devido ao isolamento social necessário para combater a pandemia. Esse número representa cerca de $80 \%$ da população estudantil mundial. Além disso, mais de 60 milhões de docentes também não podem trabalhar presencialmente em salas de aula (SEMESP, 2020).

Estudos trazem que uma das soluções foi ofertar educação continuada por meio de canais alternativos de ensino e aprendizagem, utilizando tecnologias para a mediação das aulas teóricas, como ambientes de ensino à distância e outros recursos de e-learning (qualquer intervenção educacional mediada eletronicamente e pode complementar o ensino presencial em qualquer grau). Tais alternativas se tornaram uma das principais prioridades das instituições com o intuito de reduzir ao mínimo o impacto da crise na educação. Essa premissa recebeu uma denominação própria: a educação a distância emergencial (UNESCO, 2020; Gewin, 2020; Nhantumbo, 2020; Bory, Flores \& Díaz, 2021; Reinhart et al., 2021).

Mediante esse novo panorama no processo educacional, enfatiza-se que os professores compreendam seu papel, não só no uso de tecnologias, mas permitindo que o recurso utilizado venha a ser inclusivo, de forma a atender às necessidades educacionais específicas de cada um. Não apenas durante o período de isolamento social pela pandemia, mas estudos prévios já indicavam que métodos de aprendizagem inovadores, envolvendo a utilização de plataformas e jogos digitais, podem ser utilizados como ferramentas para melhorar a qualidade de ensino em sala de aula (Granjeiro, 2019; Tupan et al., 2021).

Assim sendo, passou-se a exigir dos docentes e discentes habilidade de manejo das plataformas virtuais, estratégia essencial para minimizar o impacto no processo de interação, anteriormente, face-a-face. A implementação dessas plataformas traz múltiplas possibilidades de comunicação interpessoal, trabalho colaborativo, avaliação e autoavaliação, acesso ao processamento de informações, interação, gestão e administração educativa (Siu, Martinez \& Lao, 2018; Granjeiro et al., 2020).

A utilização de diferentes plataformas virtuais de ensino-aprendizagem possibilitou a realização de atividades síncronas (reuniões virtuais, lives, chats, entre outras), bem como assíncronas, as quais evolvem a disponibilização de vídeos e trabalhos remotos diversificados e demonstraram-se pertinentes para enfrentamento de diferentes desafios, incluindo o encurtamento das distâncias; o acesso às aulas, palestras e seminários; produções científicas; congressos virtuais, ofertando um maior leque de possibilidades de participação em diversas atividades (Sandhu \& Wolf, 2020; Moszkowicz et al., 2020).

É válido salientar que a valorização do diálogo entre os estudantes e professores, bem como o saber ouvir e refletir, mesmo que em ambiente virtual, é fundamental no processo relacional educador-educando, premissas do Modelo Dialógico de Freire. Para isso, as diversas metodologias devem ser guiadas pela qualidade dos conteúdos oferecidos, pois é fundamental a interação adequada e estimulante entre os participantes, o que pode favorecer a autonomia e requer avaliação constante da eficácia das tecnologias utilizadas (Freire, 1987; Tavares et al., 2018).

A busca pela manutenção da perspectiva crítico-reflexiva, mesmo no ensino remoto emergencial, exigiu criatividade principalmente dos docentes para que os discentes participassem ativa e dialogicamente das discussões dos conteúdos. Logo, o trabalho nesse tipo de ensino exige dos professores, além da criatividade, atenção aos horários, dedicação, elaboração de vídeos, postura profissional para essa interação e adequação do ambiente familiar (Bastos et al., 2020).

Considera-se que o sucesso das novas formas de ensino-aprendizagem depende de diversos fatores, incluindo aprendizagem ativa, interação professor-aluno, tempo das tarefas e cooperação entre eles. A aprendizagem ativa é reconhecida como estando correlacionada a um maior envolvimento dos alunos durante os cursos online que, por sua vez, contribui para uma aprendizagem online eficaz. Enquanto isso, o envolvimento do aluno é mais importante no ambiente de aprendizagem online do que na educação tradicional presencial. Estabelecer um sistema de avaliação online eficaz em um contexto do mundo real é um pré-requisito para melhorar a aprendizagem online ativa (Yan et al., 2021). 
Nesse sentido, Tecnologia Educacional (TE) representa um termo integrador ao se considerar a incorporação de diversas ciências, tecnologias e técnicas, concebendo-se como uma disciplina viva, dado o grande número de transformações a que foi exposta ao longo dos anos, como parte das mudanças resultantes do contexto; e onde ganhou valor inquestionável em tempos de pandemia (García-Leal et al, 2021).

\subsection{Educação a distância emergencial: barreiras e facilitadores}

A continuidade das atividades de ensino, pesquisa e extensão foram mantidas pelas Instituições de Ensino Superior (IES) de forma remota, mantendo o vínculo entre docentes e discentes e com vistas a atender com agilidade e efetividade as demandas da formação acadêmica. Dessa forma, professores das mais diversas áreas do saber, com ênfase na área da saúde, constituída pelas raízes práticas e vivenciais, precisaram adaptar o ensino para este novo contexto (Silva et al., 2020).

Em uma perspectiva de oportunizar a aprendizagem de forma flexível e virtual, acredita-se que é possível continuar com a metodologia do processo educacional com o apoio das tecnologias, afim de diminuir os impactos ou efeitos do isolamento social na formação de milhares de alunos. Para o enfrentamento desse novo cenário é exigido um planejamento robusto da equipe educacional, de modo a evitar uma exacerbação das desigualdades de aprendizagem dentro e entre as redes de educação (Bezerra, 2020; Castro et al., 2021).

Neste contexto, pensar sobre a redução das desigualdades é uma estratégia que pode reduzir a evasão nas universidades. Assim, algumas possibilidades precisam ser viabilizadas para aqueles que, neste momento, podem utilizar recursos tecnológicos e também aos que não dispõem de tais. Além dos recursos tecnológicos, ainda existem as especificidades relacionadas a saúde mental e a rotina dos discentes que precisam ser levadas em consideração para o aprendizado. Portanto, é importante que se estabeleça uma linha de comunicação aberta com todos os discentes, pois estes podem estar enfrentando outras situações ou aumento de demandas do trabalho, influenciando o seu rendimento acadêmico (Tantillo \& Christopher, 2020).

Embora o aprendizado online seja a única solução disponível durante a pandemia da COVID-19, a satisfação dos alunos e professores é crucial para um processo de aprendizado eficaz e bem-sucedido. A satisfação do aluno e do corpo docente pode ser definida como atitude resultante de uma avaliação da experiência educacional, instalações e serviços. A satisfação do corpo docente é definida como a percepção do processo de ensino online como eficiente, eficaz e benéfico para alunos e professores. Por outro lado, a satisfação do aluno está relacionada ao valor das experiências de aprendizagem (Weerasinghe \& Fernando, 2017; Bolliger, Inan \& Wasilik, 2014).

Do ponto de vista de alunos e professores, a aprendizagem à distância pode ter benefícios na aquisição de conhecimento especializado e aplicado e, portanto, neste aspecto, o sucesso da aprendizagem geral pode ser maior. O trabalho centrado no aluno pode ser promovido em um termo online, estimulando o ritmo individual na aprendizagem, mas as barreiras são descritas, ou seja, os limites para definir as próprias preferências. Investigações realizadas nos Emirados Árabes Unidos e França com estudantes e docentes apontaram diversos desses benefícios (Al-Samarraie et al., 2018; Rad et al., 2021; MotteSignoret et al., 2021).

Realizar o processo educacional remoto pelos professores tem como desafio a aprendizagem colaborativa valorizando o saber de cada estudante da turma e sendo mediador da sala de aula no ambiente virtual. O uso das tecnologias digitais com finalidade pedagógica tem revelado práticas de ensino motivadoras, reflexivas, diversificadas, multissensoriais, dialógicas, colaborativas, interativas, dinâmicas e mais flexíveis aos horários e espaços geográficos. Portanto, quando utilizadas de maneira coparticipante e corresponsável por docentes e discentes, as tecnologias podem qualificar as práticas de ensino, representando um meio de socializar o conhecimento e facilitar o aprendizado (Silveira \& Cogo, 2017). 
A educação a distância emergencial tem sido uma resposta válida pela resolução imediata do problema de garantir a continuidade do ensino, mas insuficiente pelo seu significado pedagógico. Uma das principais causas das limitações desta alternativa é determinada pelo insuficiente preparo dos professores para um desempenho efetivo na operação de plataformas e recursos digitais de forma a reformular o processo de ensino, o que não permite o cumprimento das condicionantes básicas para alcançar uma educação virtual de qualidade (Gianinni, 2020; Rojas \& Rossel, 2020).

Nesse repentino processo de adequação, revela-se uma inversão momentânea de papéis: educadores que antes assumiam um local de opressores passam a ocupar o local de oprimidos, necessitando aprender sobre outra dimensão do processo de ensino-aprendizagem, com o desafio de manter as premissas da educação libertadora. A imprescindibilidade do processo de capacitação para utilização dos ambientes virtuais se deve ao entendimento limitado sobre o ensino online. De modo geral, os docentes costumam ser recrutados para essa modalidade de ensino por suas especialidades e habilidade em lecionar, mas não recebem o devido treinamento para aprender conceitos teóricos sobre o ambiente virtual, o que pode intensificar o comportamento de resistência no corpo docente em relação ao ensino remoto (Gazza, 2017).

Embora os docentes reconheçam as potencialidades do uso das ferramentas tecnológicas no contexto educacional, alguns não possuem instrumentalização necessária para incorpora-las nas situações de ensino e aprendizagem, uma vez que a formação pedagógica, muitas vezes, não contempla a utilização de tecnologias digitais (Schuhmache, Alves Filho \& Schuhmacher, 2017; Silva et al., 2020).

Muitos autores relatam que dificuldades técnicas podem levar a uma experiência de aprendizagem ineficaz, mesmo utilizando o curso online mais bem elaborado. Os mesmos autores também relam que fatores relacionados à tecnologia podem impactar a satisfação de alunos e professores com a educação online, incluindo o nível de suporte técnico em que podem confiar e a facilidade de uso da infraestrutura tecnológica de seus cursos (Wingo, Ivankova \& Moss, 2017).

Diante dessas necessidades, devido à nova realidade, demandou que as IES propusessem capacitações voltadas para os docentes, com foco no manuseio desses dispositivos tecnológicos para fins pedagógicos (Silva et al., 2020; Bory, Flores \& Díaz, 2021; Cote et al., 2020).

Entretanto os desafios do ensino remoto não se fazem presentes apenas pela dificuldade de acesso e restrições aos dispositivos tecnológicos, mas também estão atrelados aos fatores motivacionais dos estudantes e professores, na necessidade de contato físico, nas relações limitadas, nas dificuldades de interação entre o docente e o discente, tais fatores implicam no processo de ensino com qualidade (Marques, 2020; Silveira et al., 2020; Gottenborg et al., 2021).

Estudo desenvolvido na China com 359 docentes avaliou como positiva a satisfação em relação ao ensino remoto e uso de tecnologias. Porém, essa satisfação dos alunos e professores pode variar já que depende de vários fatores, como conteúdo, interface do usuário, comunidade de aprendizagem e desempenho de aprendizagem, fatores esses que podem alterar diretamente o nível de motivação e satisfação dos envolvidos. A satisfação do corpo docente também impacta substancialmente os resultados do curso online (Cheng et al., 2021; Tratnik, Urh \& Jereb, 2019; Venkatesh et al., 2020).

Apesar das diferentes estratégias adotadas para o ensino remoto emergencial, é bem verdade que, na perspectiva freiriana, o distanciamento educador-educando é um outro desafio crítico do processo pedagógico. Seguindo essa perspectiva, que mais interação com os alunos é essencial para o sucesso do ensino online, pois pode reduzir a distância psicológica percebida e o estresse e fazer os alunos se sentirem apoiados em seu aprendizado online (Freire, 1987).

O ensino remoto vem desafiando os professores para novas formas de avaliação e interação com os discentes. Nesse sentido, sugere-se novas formas de interações e avaliações mais dinâmicas, um exemplo é o processo com feedbacks e interlocução entre o docente e os discentes, com diagnósticos das situações e definição de critérios, instrumentos e procedimentos adequados para o desenvolvimento das aulas (Förster et al., 2020). 
Diante dos desafios envolvendo a aplicação de práticas pedagógicas inovadoras com foco na formação inicial e continuada em saúde, deve-se observar a capacidade das instituições em possibilitar atividades pedagógicas de qualidade e a dos alunos em ter a estrutura e o apoio necessários para absorver este material, procurando minimizar as já elevadas desigualdades de aprendizado no Brasil (Granjeiro et al., 2020; Walsh et al., 2021).

Mesmo com a busca de uma dinâmica educacional mais humanista, o corpo docente se ver frente a desafios já existentes, que foram ampliados com a nova dinâmica educacional, como à carga de trabalho exaustiva, questões de envolvimento do aluno e tempo gasto na preparação de materiais de ensino. O apoio institucional é essencial para a superação dos problemas citados e tem sido recomendado por diversos autores. Este apoio poderia ser na forma de conceder tempo suficiente e incentivos compensatórios para o corpo docente (Al-Zahrani, 2015; Al-Fraihat et al., 2020; Vanderlinden, 2014; Li et al., 2021).

Mesmo com esses desafios é necessário que os docentes e gestores em educação estejam sensibilizados de que a oferta de dispositivos tecnológicos não garante educação qualificada, visto que são fundamentais ações pedagógicas, que promovam a construção do conhecimento de forma crítica e vinculada a realidade social, e imbricadas com a autonomia e cooperação dos discentes. Desta forma, os recursos tecnológicos são estratégias facilitadoras, para que as atividades de ensino sejam mantidas, assim como o vínculo entre docentes e discentes neste período excepcional da pandemia (Silveira \& Cogo, 2017; Elshami et al., 2021).

É importante examinar e refletir sobre essa experiência para melhor se preparar para a potencialidade de recorrência ou vivência de outras emergências que requerem transição imediata para o ensino e aprendizagem online. Além disso, as lições aprendidas com essa transição repentina da educação de adultos têm o potencial de transformar positivamente o ensino e a aprendizagem pós-pandemia, especialmente em programas que dependem fortemente do treinamento clínico, uma vez que foram os mais impactados desde o início da pandemia (Rad et al., 2021).

Como uma das limitações para a construção desse estudo destaca-se o número restrito e limitado de evidencias cientificas sobre a temática, considerando que ainda é uma área de abordagem recente. Assim sendo, este estudo visa contribuir com a identificação de lacunas que necessitam ser preenchidas, instigando o meio acadêmico-cientifico ao desenvolvimento de novas pesquisas e investigações.

\section{Considerações Finais}

Este estudo de revisão destacou a abordagem recente na literatura sobre a Docência do Ensino Superior em saúde durante a pandemia da Covid-19, destacando a escassez de produções científicas principalmente a nível de Brasil.

O atual cenário de saúde pública exige das Instituições de Ensino Superior um processo de transformação, adaptação e exercício da criatividade para lidar com o ensino remoto emergencial e o uso das tecnologias digitais. Entretanto é preciso estar atento as desigualdades e vulnerabilidades que podem surgir com esse processo, exigindo uma atenção diferenciada, uma vez que pessoas com classes sociais desfavoráveis podem ser afetadas.

Apesar de ser uma alternativa para o enfrentamento da pandemia e garantia do processo de formação, deve-se atentar para os desafios inerentes a qualquer situação. O ensino precisa ser organizado de forma atrativa, flexível e acessível, bem como as instituições de ensino precisam apoiar e capacitar discentes e docentes.

\section{Referências}

Al-Fraihat, D., Joy, M., Masa'deh, R., \& Sinclair, J. (2020). Evaluating E-learning systems success: an empirical study. Comput Human Behav, 102:67-86. 
Al-Samarraie, H. Theng, B. K. Alzahrani, A. I., \& Alalwan, N. (2018). E-learning continuance satisfaction in higher education: a unified perspective from instructors and students. Stud High Educ, 43(11): 2003-2019.

AL-Zahrani, A. M. (2015). Faculty satisfaction with online teaching in Saudi Arabia's higher education institutions. International Journal of Instructional Technology and Distance Learning, 12(4): 17-28.

Bastos, M. C., Canavarro, D. A., Campos, L. M., Schulz, R. S., Santos, J. B., \& Santos, C. F. (2020). Ensino remoto emergencial na graduação em enfermagem: relato de Experiência na covid-19. REME, 24: e-1335.

Bezerra, I. M. (2020). State of the art of nursing education and the challenges to use remote technologies in the time of coronavirus pandemic. Rev Bras Crescimento Desenvolv Hum, 30(1): 141-7.

Bolliger, D. U., Inan, F. A., Wasilik, O. (2014). Development and validation of the online instructor satisfaction measure (OISM). J Educ Technol Soc, 17(2): $183-195$.

Bory, E. J. P., Flores, L. G. A, \& Díaz, K. G. (2021). Innovación emergente para la COVID-19: taller virtual sobre el uso educativo de la plataforma Moodle. Revista Cubana de Informática Médica, 13(1): e438.

Brasil. Ministério da Educação. (2020). Portaria $n^{\circ} 343$ de 17 de março de 2020. Dispõe sobre a substituição das aulas presenciais por aulas em meios digitais enquanto durar a situação de pandemia do Novo Coronavírus. http://www.in.gov.br/en/web/dou/-/portaria-n-343-de-17-de-marco-de-2020-248564376

Brasil. Ministério da Saúde. (2021). Coronavírus (COVID-19). https://news.google.com/covid19/map?hl=ptBR\&mid=\%2Fm\%2F015fr\& $\mathrm{gl}=\mathrm{BR} \&$ ceid=BR\%3Apt-419

Brasil. Ministério da Saúde. (2020). Coronavírus Brasil: síntese de casos, óbitos, incidência e mortalidade. https://covid.saude.gov.br/

Carvalho, M. S., Lima, L. D., \& Coeli, C. M. (2020). Ciência em tempos de pandemia. Cad. Saúde Pública, 36(4): e00055520.

Castaman, A. S., \& Rodrigues, R. A. (2020). Educação a Distância na crise COVID -19: um relato de experiência. Research, Society and Development, 9(6): $1-26$.

Castro, Í. F. A.., Sousa, F. C., Cipriano, T. H. A. S., Costa, F. G., Castro, K. S., Costa, V. S., Pires, G. R., \& Narzetti, V. R. (2021). Residência Pedagógica e ensino a distância: desafios e perspectivas na visão dos professores da educação básica participantes do programa. Pesquisa, Sociedade e Desenvolvimento, 10(11): e75101119359.

Cespedes, M. S., \& Souza, J. C. P. (2020). Coronavirus: a clinical update of Covid-19. Rev. Assoc. Med. Bras, 66(2): 16-123.

Cheng, X., Chan, L. K., Pan, S., Cai, H., Li, Y., \& Yang, X. (2021). Gross Anatomy Education in China during the Covid-19 Pandemic: A National Survey. Anat Sci Educ 14:8-18.

Cote, M. P., Donne, E. M., Hoover, B. D., \& Thormodson, E. K. (2020). Teaching Instructional Technological Change to Medical School Faculty: A COVID19 Case Study. Medical Reference Services Quarterly, 39(4): 406-41.

Elshami, W., Taha, M. H., Abuzaid, M., Saravanan, C., Al Kawas, S., Abdalla, M. E. (2021). Satisfaction with online learning in the new normal: perspective of students and faculty at medical and health sciences colleges, Medical Education Online, 26(1): 1920090.

Förster, C., Eismann-Schweimler, J., Stengel, S., Bischoff, M., Fuchs, M., Graf von Luckner, A., Ledig, T., Barzel, A., Maun, A., Joos, S., Szecsenyi, J., \& Schwill, S. (2020). Opportunities and challenges of e-learning in vocational training in General Practice - a project report about implementing digital formats in the KWBW-Verbundweiterbildung. Journal for Medical Education, 37(7), 2020.

Freire, P. (1987). Pedagogia do oprimido. Rio de Janeiro: Paz e Terra.

García-Leal, M., Medrano-Rodríguez, H., Vázquez-Acevedo, J. A., Romero-Rojas, J. C., \& Berrún-Castañón, L. N. (2021). Experiencias docentes del uso de la tecnología educativa en el marco de la pandemia por COVID-19. Revista Informacion Cientifica, 100(2).

Gazza, E. A. (2017). The experience of teaching online in nursing education. J Nurs Educ, 56(6): 343-9.

Gewin, V. (2020). Five tips for moving teaching online as COVID-19 takes hold. Nature, 580(7802): 295-6.

Giannini, S. (2020). COVID-19 y educación superior: de los efectos inmediatos al día después. Revista Latinoamericana de Educación Comparada, 11(17): 157.

Gottenborg, E., Yu, A., Naderi, R. Keniston, A., McBeth, L., Morrison, K., Schwartz, D., \& Burden, M. (2021). COVID-19's impact on faculty and staff at a School of Medicine in the US: what is the blueprint for the future? BMC Health Services Research, 21(395).

Granjeiro, E, M., Musse, J. O., Peixoto, T. M., Nunes, I. V., Soares, I. M. S. C., I. C. O., Silva, I. C. O., Carvalho, T. B., \& Dias, Y. O. (2020). Estratégias de ensino à distância para a educação interprofissional em saúde frente à pandemia COVID-19. REVISA, 9(1): 591-602.

Granjeiro, E. M. (2019). Research-based teaching-learning method: a strategy to motivate and engage students in human physiology classes. Advances in Physiology Education, 43: 553-556.

Li, W., Gillies, R., He, M., Wu, C., Liu, S., Gong, Z., \& Sun, H. (2021). Barriers and facilitators to online medical and nursing education during the COVID-19 pandemic: perspectives from international students from low- and middle-income countries and their teaching staff. Hum Resour Health, 19(64).

Marques, R. (2020). A ressignificacao da educacao e o processo de ensino e aprendizagem no contexto de pandemia da COVID-19. Boletim De Conjuntura, 3(7): $1-18$. 
Melnyk, B. M., \& Fineout-Overholt, E. (2005). Making the case for evidence-based practice. In: Evidence-based practice in nursing \& healthcare: a guide to best practice. Philadelphia: Lippincot Williams \& Wilkins.

Mendes, K. D. S., Silveira, R. C. C. P., \& Galvão, C. M. (2008). Revisão integrativa: método de pesquisa para a incorporação de evidências na saúde e na enfermagem. Texto Contexto Enferm, 17(4): 758-764.

Mojica, C. R., \& Morales, C. M. M. (2020). Pandemia COVID-19, la nueva emergencia sanitaria de preocupación internacional: una revisión. Semergen, 46(1): 65-77.

Moszkowicz, D., Duboc, H., Dubertret, C., Roux, D., \& Bretagnol, F. (2020). Daily medica education for confined students during COVID-19 pandemic: a simple videoconference solution. Clin Anat, 33(6): 927-928.

Motte-Signoret, E., Labbé, A., Benoist, G., Linglart, A., Gajdos, V., \& Lapillonne, A. (2021). Perception of medical education by learners and teachers during the COVID-19 pandemic: a cross- sectional survey of online teaching. Medical Education Online, $26(1): 1919042$.

Nhantumbo, T. L. (2020). Capacidade de resposta das instituicoes educacionais no processo de ensino-aprendizagem face a pandemia de COVID19: impasses e desafios. Rev EDUCAmazonia, 25(2): 556-71.

Rad, F. A., Otaki, F., Baqain, Z., Zary, N., \& Al-Halabi, M. (2021). Rapid transition to distance learning due to COVID-19: Perceptions of postgraduate dental learners and instructors. PLoS ONE, 16(2): e0246584.

Reinhart, A., Malzkorn, B., Döing, C., Beyer, I., Jünger, J., \& Bosse, H. M. (2021). Undergraduate medical education amid COVID-19: a qualitative analysis of enablers and barriers to acquiring competencies in distant learning using focus groups. Medical Education Online, $26(1): 1940765$.

Rojas, C. F. C., \& Rosell, R. C. A. (2020). Desafíos de la educación virtual en Odontología en tiempos de pandemia COVID-19. Rev Cubana Estomatol, 57(3): 3341-3344

Sandhu, P., \& Wolf, M. (2020). The impact of COVID-19 on the undergraduate medical curriculum. Med Educ Online, 25(1): 1764740.

Schuhmacher, V. R., Alves Filho, J. P., \& Schuhmacher, E. (2017). As barreiras da pratica docente no uso das tecnologias de informacao e comunicacao. Cienc Educ, 23(3): 563-566.;

SEMESP. (2020). Estudo/Efeitos da pandemia na educação superior brasileira. https://www.semesp.org.br/pesquisas/estudo-efeitos-da-pandemianaeducacaosuperior-brasileira/

Silva, A.F., Estrela, F. M., Lima, N. S., \& Abreu, C. T. A. (2020). Saude mental de docentes universitarios em tempos de pandemia. Physis, $30(2)$ : e300216.

Silveira, A., Santos, N. O., Wilhelm, L. A., Soccol, K. L. S., Tisott, Z. L., \& Prates, L. A. (2020). Estratégias e desafios do ensino remoto na enfermagem. Enferm. Foco, 11(5): 98-103.

Silveira, M. S., \& Cogo, A. L. (2017). Contribuicoes das tecnologias educacionais digitais no ensino de habilidades de enfermagem: revisao integrativa. Rev Gaucha Enferm, 38(2): e66204.

Siu, J. A. L., Martinez, A. P., \& Lao, J. M. I. (2018). Plataforma interactiva para la integración em el proceso de extensión universitária. MEDISAN, 22(4).

Sousa, A. J. M., Torres, A. A., Araujo, M. M., Dias, F. I. C. R., Montelo, E. S., \& Nogueira, F. J. S. (2020). Atenção Primária à Saúde e Covid-19: uma revisão integrativa. Cadernos Esp Ceará, 14(1): 45-52.

Tantillo, L., \& Christopher, R. (2020). Transforming graduate nursing education during an era of social distancing: Tools from the field. Nurse Educ Today, 92(esp): 104472 .

Tavares,A. P. C., Leite, B. S., Silveira, I. A., Santos, T. D., Brito, W. A. P, \& Camacho, A. C. L. F. (2018). Analysis of Brazilian publications on distance education in nursing: integrative review. Rev Bras Enferm, 71(1): 214-22.

The World Bank. (2020). Políticas Educacionais na Pandemia da COVID-19: o que o Brasil pode Aprender com o Resto do Mundo? https://www.worldbank.org/pt/country/brazil/publication/brazileducation-policy-covid-19-coronavirus-pandemic

Tratnik, A., Urh, M., \& Jereb, E. (2019). Student satisfaction with an online and a face-to-face business English course in a higher education context. Innov Educ Teach Int, 56(1): 36-45.

Tupan, L. F. S., Nunes, G. C. S., Mincache, A. J., \& Souza, A. O. (2021). Perspectivas de professores de Física por meio do ensino à distância durante a pandemia COVID-19. Pesquisa, Sociedade e Desenvolvimento, 10(11): e27101119293.

UNESCO. Organização das Nações Unidas para a Educação, a Ciência e a Cultura. (2020) ¿Cómo estás aprendiendo durante la pandemia de COVID-19? Impacto COVID-19 en la educación. https://es.unesco.org/covid19/ educationresponse

Vanderlinden, K. (2014). Blended Learning as Transformational Institutional Learning. New Dir High Educ, 2014(165): 75-85.

Venkatesh, S., Rao, Y. K., Nagaraja, H., Woolley, T., Alele, F.O., \& Malau-Aduli, B. S. (2020). Factors influencing medical students' experiences and satisfaction with blended Integrated E-Learning. Med Princ Pract, 29(4): 396-402.

Walsh, L. L., Arango-Caro, S., Wester, E. R., \& Callis-Duehl, K. (2021). Training Faculty as an Institutional Response to COVID-19 Emergency Remote Teaching Supported by Data. Life Sciences Education, 20(34):1-11.

Weerasinghe, I. S., \& Fernando, R. L. (2017). Students' satisfaction in higher education. American Journal of Educational Research, 5(5): 533-9. 
Research, Society and Development, v. 10, n. 15, e173101522828, 2021

(CC BY 4.0) | ISSN 2525-3409 | DOI: http://dx.doi.org/10.33448/rsd-v10i15.22828

Wingo, N. P., Ivankova, N. V., \& Moss, J. A. (2017). Faculty perceptions about teaching online: exploring the literature using the technology acceptance model as an organizing framework. Online Learning $J, 21(1)$.

Yan, Y., Cheng, X., Zhou, C., Yang, X., \& Li, Y. (2021). The perceptions of anatomy teachers for different majors during the COVID-19 pandemic: a national Chinese survey. Medical Education Online, 26(1): 1897267, 2021. 